\title{
Zero Temperature Magnetic Frustration in Nona-Membered $s=3 / 2$ Spin Rings with Bond Defect
}

\author{
M. Antkowiak, P. KozŁowski and G. Kamieniarz* \\ A. Mickiewicz University, Faculty of Physics, Umultowska 85, 61-614 Poznań, Poland
}

\begin{abstract}
Zero-temperature limits of the local and global thermodynamic quantities in the nine-membered antiferromagnetic $s=3 / 2$ spin ring are investigated by means of numerical exact diagonalization. An anisotropic Heisenberg model with tunable bond defect reflecting continuously varying topology (from closed to open ring) is exploited. The frustrated and non-frustrated phases are identified in the ground-state phase diagram determined by a bond-defect strength and magnetic field. Near the phase boundaries significance of the thermal fluctuations affecting the estimates of the local magnetic quantities found earlier at $T=1 \mathrm{~K}$ is revealed. For the global quantities the effects of thermal fluctuations are found much weaker. A sequence of the local magnetic moments is analysed and their experimental verification at the edges of the non-frustrated and within the entire frustrated phase is suggested at sufficiently low temperature.
\end{abstract}

PACS: 75.40.Cx, 75.10.Jm

\section{Introduction}

Molecular nanomagnets based on transition metal ions have been very intensively investigated [1]. Their popularity is mostly due to the fact that quantum phenomena characteristic for a single molecule (like, e.g., quantum tunneling or step like field dependence of magnetization) can be observed in bulk samples. It is possible because nanomolecules are magnetically shielded from each other by organic ligands and the dominant interactions are those within the molecule. There are also expectations that this kind of materials may find application in quantum computing [2-5] and information storage [6].

A large family of molecular nanomagnets comprises ring-shaped molecules. Most of them contain even number of antiferromagnetically interacting ions. Only recently the first odd membered antiferromagnetic molecules have been reported [7-9]. They are especially interesting because of magnetic frustration which is expected to appear in this kind of materials.

In our recent paper [10] we analyzed frustration signatures of a nine-membered $s=3 / 2$ spin ring with changing topology determined by a single bond defect. The ring can be considered as a model of a hypothetical nine-membered chromium molecule $\mathrm{Cr}_{9}$ with 8 nearest neighbour couplings $J$ and one coupling $J^{\prime}$ between the sites $i=1$ and $i=9$, defining the bond defect $\alpha=J^{\prime} / J$. For $\alpha=0$ one gets magnetically open ring and for $\alpha=1$ a perfect symmetric ring is obtained. In the ring molecules synthesized so far, the $\mathrm{Cr}$ (III) ions are linked by one fluorine and two carboxylate bridges responsible for superexchange. Experimental realization of our model in this type of molecules requires a single modification of the bridges involved and seems feasible to achieve [11].

\footnotetext{
* corresponding author; e-mail: gjk@amu.edu.pl
}

In the model in question with applied magnetic field $B=5 \mathrm{~T}$ we found [10] two limiting values $\alpha_{\mathrm{c}}=0.32$ and $\alpha_{\mathrm{s}}=1$ corresponding to the border between the unfrustrated and frustrated ground state, and the limit of the uniform ring, respectively. We note that in Figs. 7-10 of Ref. [10] the curves calculated for $T=1 \mathrm{~K}$ and plotted as a function of $\alpha$ are continuous, smooth and intersect one another at $\alpha_{\mathrm{s}}=1$. In addition, the second moment of the zero-field magnetization in the unfrustrated region $\alpha<0.32$ (Fig. 10 in Ref. [10]) is far from the expected $T=0$ value $9 / 4$, whereas in the frustrated region agrees with the $T=0$ value $1 / 4$.

In this paper we focus on zero temperature quantities in order to separate the quantum effects from the thermal fluctuations. Before [10] the characteristics at $T=1 \mathrm{~K}$ were extracted by the quantum transfer method which fails in the limit $T=0$. Only the total magnetization, as a constant of motion, was calculated for $T=0$ and $T=0.1 \mathrm{~K}$ by a simpler variant of the numerical exact diagonalization. Using this technique oriented towards calculation of the local quantities and implemented on large parallel computers [12, 13], we would like to answer the following questions: (a) are the quantum effects always dominant over the thermal fluctuations at $T=1 \mathrm{~K}$ ? (b) which values of the defect $\alpha$ are preferred for experimental verification of the local magnetizations in the corresponding envisaged real system?

As to the latter, in the NMR experiment [14] conducted for the non-frustrated ring $\mathrm{Cr}_{7} \mathrm{Cd}$ at $T=1.5 \mathrm{~K}$, where $\mathrm{Cd}(\mathrm{II})$ ion brings a nonmagnetic impurity which corresponds to a seven-numbered $s=3 / 2$ spin ring with $\alpha=0$, the local moments localised on the $\mathrm{Cr}$ (III) sites were measured with accuracy of the order of $10 \%$.

\section{Results}

The physics of the chromium ring can be well described by the Heisenberg Hamiltonian with the uniaxial single- 
-ion anisotropy expressed by Eq. (1) in Ref. [10]. The values assumed are $g=1.98$, the coupling $J / k_{\mathrm{B}}=-16.6 \mathrm{~K}$ and $D / k_{\mathrm{B}}=-0.34 \mathrm{~K}$, which are typical of $\mathrm{Cr}$ molecules $[15,16]$, and the field $B$ is applied along the $z$ axis.

Following Ref. [10] the following local quantities $m_{j}=$ $\left\langle s_{j}^{z}\right\rangle, \delta m_{j}=\left\langle\left(s_{j}^{z}\right)^{2}\right\rangle-\left\langle s_{j}^{z}\right\rangle^{2}, C_{i j}=\left\langle s_{i}^{z} s_{j}^{z}\right\rangle, \delta C_{i j}=\left\langle s_{i}^{z} s_{j}^{z}\right\rangle-$ $\left\langle s_{i}^{z}\right\rangle\left\langle s_{j}^{z}\right\rangle$ are calculated for $1 \leq i \leq 5$.

Quantities dependent on two local spins are considered only for nearest neighbors (NN, i.e. $j=i+1$ ). The quantities for $i>5$ are related to those for $i \leq 4$ by symmetry. Besides, also global quantities (the first and the second moment of magnetization denoted by $M$ and $M_{2}$ ) are calculated for $B=5 \mathrm{~T}$ and $B=0$, respectively. Interestingly, the values $M$ and $M_{2}$ can distinguish the area of frustrated and unfrustrated spins [10].

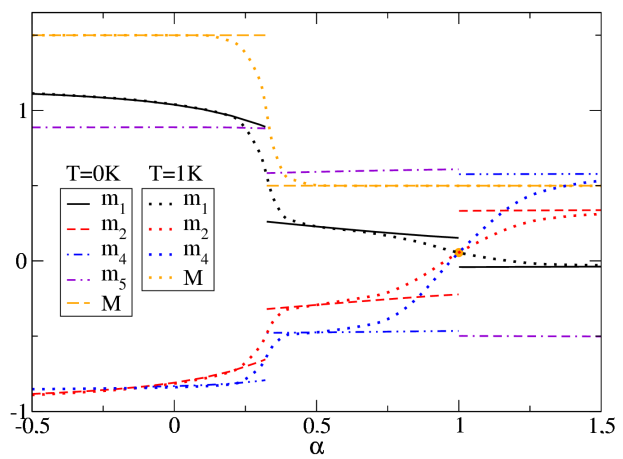

Fig. 1. Selected local and global magnetizations for $T=0$ and $T=1 \mathrm{~K}$ calculated in the field $B=5 \mathrm{~T}$.

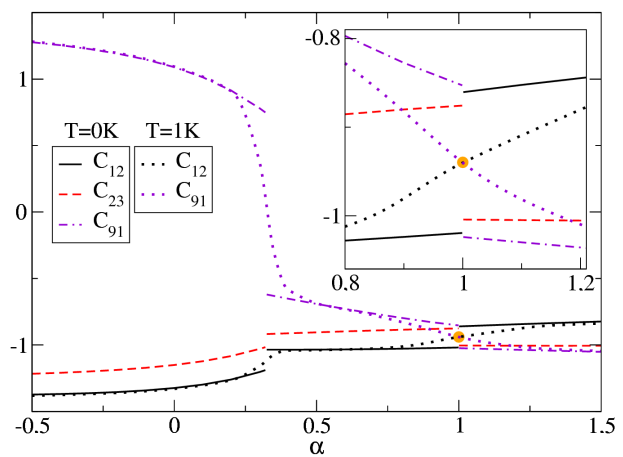

Fig. 2. Selected NN spin correlations $C_{i j}$ in the field $B=5 \mathrm{~T}$. In the inset $C_{91}$ and $C_{12}$ are compared around $\alpha=1$ for $T=0$ and $T=1 \mathrm{~K}$, demonstrating thermal effects.

In Figs. 1, 2 and 3 the local quantities calculated in the ground state are presented as functions of $\alpha$ for $B=5 \mathrm{~T}$. All of them display discontinuities at the limiting values $\alpha_{\mathrm{c}}(B)=0.32$ and $\alpha_{\mathrm{s}}=1$. The discontinuities correspond to the energy level crossings in the ground state. The behaviour in the vicinity of the symmetric point $\alpha_{\mathrm{s}}=1$, denoted by the full circle, is striking both in the presence and in the absence of magnetic field (Figs. 1-4). The $T=0$ curves do not approach each other and display a

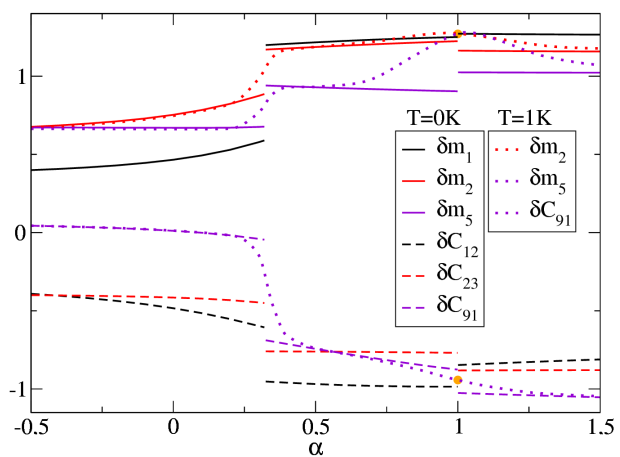

Fig. 3. Local spin fluctuations $\delta m_{j}$ and correlations of fluctuations $\delta C_{i j}$ versus $\alpha$ for $B=5 \mathrm{~T}$ at $T=0$ and $T=1 \mathrm{~K}$.

step. The correlations $C_{19}$ are clearly ferromagnetic in the unfrustrated phase $\alpha<0.32$ (Figs. 2, 4).

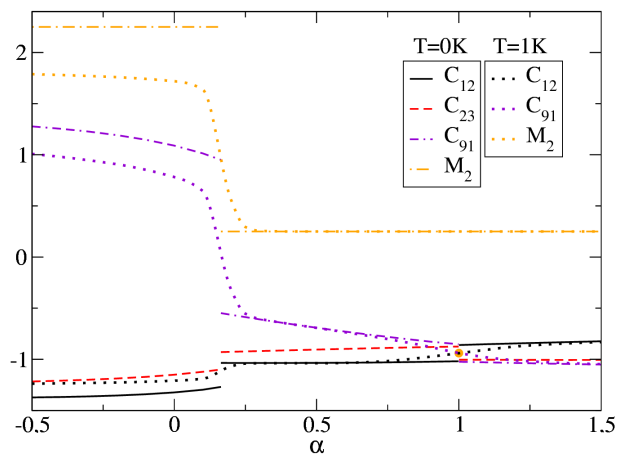

Fig. 4. Selected NN spin correlations $C_{i j}$ and the second moment $M_{2}$ as a function of $\alpha$ for $T=0, T=1 \mathrm{~K}$ and $B=0$

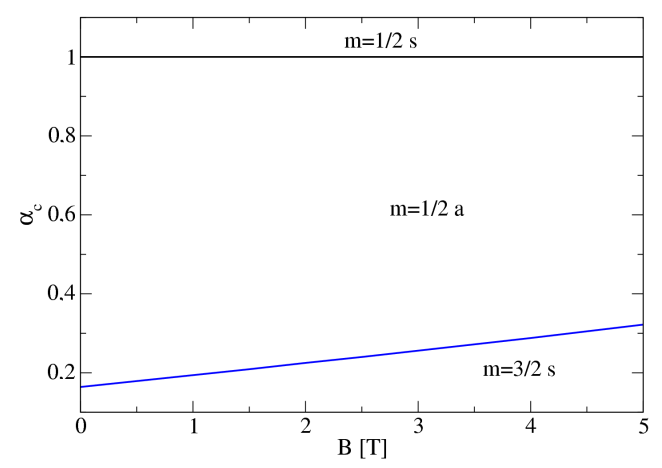

Fig. 5. The ground state $(\alpha, B)$ phase diagram.

We expect that the behaviour of the local quantities illustrated in Figs. 1, 2, 3 and 4 is typical and can be found also for fields different from 0 and $5 \mathrm{~T}$. Indeed, in the phase diagram shown in Fig. 5 the unfrustrated phase is separated by a continuous line $\alpha_{\mathrm{c}}(B)$ from the frustrated phase and the special point $\alpha=1$ detaches 
the symmetric and antysymmetric $m=1 / 2$ frustrated phases irrespective of the value of $B$.

At zero temperature the global first and second moments ( $M$ and $M_{2}$, respectively) sharply distinguish the frustrated and unfrustrated phase due to the jumps appearing for $\alpha=0.32$ (Fig. 1 and Fig. 4). The $T=0$ value $M_{2}=9 / 4$ of the zero-field second moment remains constant in the unfrustrated phase (Fig. 4), whereas that for $T=1 \mathrm{~K}$ is significantly reduced and weakly dependent on $\alpha$. In the frustrated phase both curves nearly coincide so that the second moment is affected by thermal fluctuations only in the unfrustrated phase. In the case of the first moment at $T=1 \mathrm{~K}$, thermal fluctuations are significant only near the critical value $\alpha=0.32$.

The analysis of the local quantities will be concentrated on the local magnetizations $m_{i}$ in view of their anticipated experimental verification. We assume that the experimental accuracy is of the order of $10 \%$ which was reached [14] at $T=1.5 \mathrm{~K}$. Previous results at $T=1 \mathrm{~K}$ (Fig. 7 in Ref. [10]) suggest that for $\alpha \leq 0.2$ only $m_{1}$ can be distinguished from the pairs $\left(m_{3}, m_{5}\right)$ and $\left(m_{2}, m_{4}\right)$, whereas in the frustrated region all the local magnetizations are well separated for $0.4 \leq \alpha \leq 0.8$ and for $\alpha>1$.1. The $T=0$ counterpart of these results (Fig. 1) allows us to expand the domain of experimental verification of the local magnetizations. In the unfrustrated region the discrimination of the local moments is only slightly increased, whereas all the local magnetizations are distinct enough to be distinguished in the entire frustrated region provided that sufficiently low temperatures are reached.

Comparison of the local magnetizations at $T=0$ and $T=1 \mathrm{~K}$ confirms the same sequence of $m_{i}$ values along the ring so that we expect that it will be preserved for all $T<1 \mathrm{~K}$. We predict that in the unfrustrated region the absolute value of $m_{1}$ is the largest, whereas for $0.32<$ $\alpha<1$ the largest is $m_{5}$ and for $\alpha>1$ the largest is $m_{4}$ while $m_{1}$ nearly vanishes. We also note that the low-temperature local spin fluctuations (Fig. 3) out of the phase boundaries are mainly of quantum nature.

\section{Conclusions}

The ground-state phase diagram is worked out in the two-parameter space determined by the bond defect $\alpha$ and magnetic field. The global and local magnetic quantities, calculated at $T=0$ as a function of $\alpha$, undergo discontinuous changes for the limiting values $\alpha=0.32$ and $\alpha=1$ which correspond to the phase boundaries.

The local thermal averages evaluated earlier at $T=$ $1 \mathrm{~K}$ are considerably distinct from the $T=0$ predictions in the close vicinity of the transition between the non-frustrated to the frustrated phase and around $\alpha=1$. As to the global quantities, $M$ is stable against thermal fluctuations, whereas $M_{2}$ is not affected in the frustrated region.

In the field $B=5 \mathrm{~T}$ all the values of the local magnetizations differ significantly in the frustrated region and can probably be verified experimentally for sufficiently low temperatures. In the non-frustrated region, only in the limited area $\alpha>0.2$ some of them are different enough to be discriminated experimentally.

\section{Acknowledgments}

We acknowledge a support from the MNiSW grant No. 290438 and an access to the platforms of the Supercomputing and Networking Center in Poznań and the Academic Computer Center in Gdańsk (Poland).

\section{References}

[1] D. Gatteschi, R. Sessoli, J. Villain, Molecular Nanomagnets, Oxford University Press, Oxford 2006.

[2] A. Ardavan, O. Rival, J.J.L. Morton, S.J. Blundell, A.M. Tyryshkin, G.A. Timco, R.E.P. Winpenny, Phys. Rev. Lett. 98, 057201 (2007).

[3] J. Lehmann, A. Gaita-Ariño, E. Coronado, D. Loss, Nature Nanotech. 2, 312 (2007).

[4] G.A. Timco, S. Carretta, F. Troiani, F. Tuna, R.J. Pritchard, Ch.A. Muryn, E.J.L. McInnes, A. Ghirri, A. Candini, P. Santini, G. Amoretti, M. Affronte, R.E.P. Winpenny, Nature Nanotech. 4, 173 (2009).

[5] B. Georgeot, F. Mila, Phys. Rev. Lett. 104, 200502 (2010).

[6] M. Mannini, F. Pineider, Ph. Sainctavit, Ch. Danieli, E. Otero, C. Sciancalepore, A.M. Talarico, M.-A. Arrio, A. Cornia, D. Gatteschi, R. Sessoli, Nature Mat. 8, 194 (2009).

[7] O. Cador, D. Gatteschi, R. Sessoli, A.-L. Barra, G.A. Timco, R.E.P. Winpenny, J. Magn. Magn. Mater. 290-291, 55 (2005).

[8] H.C. Yao, J.J. Wang, Y.S. Ma, O. Waldmann, W.X. Du, Y. Song, Y.Z. Li, L.M. Zheng, S. Decurtins, X.Q. Xin, Chem. Commun., 1745 (2006).

[9] N. Hoshino, M. Nakano, H. Nojiri, W. Wernsdorfer, H. Oshio, J. Am. Chem. Soc. 131, 15100 (2009).

[10] P. Kozłowski, M. Antkowiak, G. Kamieniarz, J. Nanopart. Res. 13, 6093 (2011).

[11] R.E.P. Winpenny, private communication.

[12] A. CaramicoD'Auria, U. Esposito, F. Esposito, G. Kamieniarz, R. Matysiak, J. Phys., Condens. Matter 13, 2017 (2001).

[13] G. Kamieniarz, P. Kozłowski, G. Musiał, W. Florek, M. Antkowiak, M. Haglauer, A. Caramico D'Auria, F. Esposito, Inorg. Chim. Acta 361, 3690 (2008).

[14] E. Micotti, Y. Furukawa, K. Kumagai, S. Carretta, A. Lascialfari, F. Borsa, G.A. Timco, R.E.P. Winpenny, Phys. Rev. Lett. 97, 267204 (2006).

[15] P. Kozłowski, G. Kamieniarz, M. Antkowiak, F. Tuna, G.A. Timco, R.E.P. Winpenny, Polyhedron 28, 1852 (2009).

[16] M. Antkowiak, P. Kozłowski, G. Musiał, W. Florek, G. Kamieniarz, F. Esposito, Acta Phys. Pol. A 118, 965 (2010). 